\title{
Direct detection and measurement of wall shear stress using a filamentous bio-nanoparticle
}

\author{
Daniela P. Lobo ${ }^{1}$, Alan M. Wemyss ${ }^{1,2}$, David J. Smith ${ }^{3}$, Anne Straube ${ }^{4}$, Kai B. Betteridge ${ }^{5}$, Andrew H. J. Salmon ${ }^{5}$, \\ Rebecca R. Foster ${ }^{6}$, Hesham E. Elhegni ${ }^{6}$, Simon C. Satchell ${ }^{6}$, Haydn A. Little ${ }^{7}$, Raúl Pacheco-Gómez ${ }^{8}$, \\ Mark J. Simmons ${ }^{9}$, Matthew R. Hicks ${ }^{8}$, David O. Bates ${ }^{10}$, Alison Rodger ${ }^{1}(\varangle)$, Timothy R. Dafforn ${ }^{8}$, and \\ Kenton P. Arkill ${ }^{11}$ \\ ${ }^{1}$ Department of Chemistry and Warwick Analytical Science Centre, University of Warwick, Coventry CV4 7AL, UK \\ ${ }^{2}$ MOAC Doctoral Training Centre, University of Warwick, Coventry CV4 7AL, UK \\ ${ }^{3}$ Mathematics, University of Birmingham, Edgbaston, Birmingham, West Midlands B15 2TT, UK \\ ${ }^{4}$ Centre for Mechanochemical Cell Biology, Warwick Medical School, University of Warwick, Coventry CV4 7AL, UK \\ ${ }^{5}$ Physiology and Pharmacology, University of Bristol, University Walk, Bristol BS8 1TD, UK \\ ${ }^{6}$ Clinical Sciences, Whitson Street, University of Bristol, Bristol BS1 3NY, UK \\ ${ }^{7}$ School of Chemistry, University of Birmingham, Edgbaston, Birmingham, West Midlands B15 2TT, UK \\ ${ }^{8}$ Biosciences, University of Birmingham, Edgbaston, Birmingham, West Midlands B15 2TT, UK \\ ${ }^{9}$ Chemical Engineering, University of Birmingham, Edgbaston, Birmingham, West Midlands B15 2TT, UK \\ ${ }^{10}$ School of Medicine, University of Nottingham, Queen's Medical Centre, Nottingham NG2 7UH, UK \\ ${ }^{11}$ Biochemistry, University of Bristol, University Walk, Bristol BS8 1TD, UK
}

Received: 19 February 2015

Revised: 4 June 2015

Accepted: 8 June 2015

(C) Tsinghua University Press and Springer-Verlag Berlin Heidelberg 2015

\section{KEYWORDS}

microfluidics, nanoparticle, M13 bacteriophage, wall shear stress, fluorescent microscopy

\begin{abstract}
The wall shear stress (WSS) that a moving fluid exerts on a surface affects many processes including those relating to vascular function. WSS plays an important role in normal physiology (e.g. angiogenesis) and affects the microvasculature's primary function of molecular transport. Points of fluctuating WSS show abnormalities in a number of diseases; however, there is no established technique for measuring WSS directly in physiological systems. All current methods rely on estimates obtained from measured velocity gradients in bulk flow data. In this work, we report a nanosensor that can directly measure WSS in microfluidic chambers with sub-micron spatial resolution by using a specific type of virus, the bacteriophage M13, which has been fluorescently labeled and anchored to a surface. It is demonstrated that the nanosensor can be calibrated and adapted for biological tissue, revealing WSS in micro-domains of cells that cannot be calculated accurately from bulk flow measurements. This method lends itself to a platform applicable to many applications in biology and microfluidics.
\end{abstract}

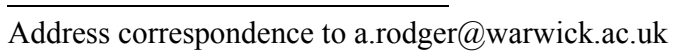




\section{Introduction}

The interaction between flowing liquids and solid surfaces, such as blood and endothelial cells, affects the function of both the surface and liquid. The force per unit area that a moving fluid exerts parallel to a surface or wall is known as the wall shear stress (WSS). This parameter affects cellular and biochemical reactions and is therefore important for a range of fields, including microfluidic system design [1], and in understanding disease states of the vascular system [2]. WSS plays an important role in normal physiology and affects the microvasculature's primary function of molecular transport [3-7]. Points of fluctuating WSS show abnormalities in diseases such as atherosclerosis, diabetes, and cancer. Measuring WSS directly is currently possible only on a relatively large scale by techniques such as microelectromechanical sensors [8] and quasi-direct methods such as deformable micropillars (a micropillar device is $\sim 100 \mu \mathrm{m}$ tall) $[9,10]$. There is no established technique for measuring WSS directly in physiological systems. All methods rely on the estimation of WSS by using velocity gradients measured close to the wall, the fluid rheology, and the assumption of a boundary condition at the surface (e.g. the Dirichlet no-slip condition). Such methods are unreliable when applied to the circulatory system, which exhibits pulsatile three-dimensional flow containing fluid particulates and has elastic walls of varying geometries. Thus, development of new methods to measure WSS is important.

In the vasculature, the velocity gradient at the wall is currently measured by bulk flow techniques such as micro-particle image velocimetry ( $\mu$ PIV) [11] to calculate the velocity gradient at the wall by making assumptions including uniformity of flow and the surface. Such an average measurement may hide variability critical to the understanding of disease states in complex, dynamic, and variable systems such as a blood vessel. $\mu$ PIV methods struggle with the complex nature of blood and are limited by the particle size used (as well as viable flow ranges). Furthermore, interpreting experimental data is dependent on the computational models (and associated assumptions) used to determine WSS in medicine [12].

Any fluid moving along a solid boundary induces a shear stress on that boundary. For a Newtonian fluid in laminar flow, such as blood plasma, the WSS is

$$
\mathrm{WSS}=\left.\eta \frac{\partial V}{\partial y}\right|_{0}
$$

where $\eta$ is the dynamic viscosity of the fluid, $V$ is its velocity, and $y$ is the height above the boundary. The horizontal axis refers to WSS as calculated from a model of laminar flow in a smooth rectangular channel (numerical values taken from manufacturer's data). This quantity is a "nominal" value because it does not correct for local variations such as roughness over the surfaces of cells.

Reported here is a strategy for measuring WSS by using labeled M13 bacteriophage (M13) as a microfluidic reporter. Previous work has reported its use as a scaffold for nanowires [13-15] and fibres $[16,17]$, as a biosensor [18], in drug delivery [19], as a cancer marker [20], and in the detection of biological molecules [18]. M13 is a long $(\sim 900 \mathrm{~nm})$ and thin $(\sim 7 \mathrm{~nm})$ semi-rigid construct whose persistence length is $\sim 1,400 \mathrm{~nm}$ [21]. The alignment in flow and spectroscopy of this filamentous particle has recently been exploited in our previous work for a variety of diagnostic assays [22]. We have generated chemical modification protocols to label M13 with fluorophores that provide a suite of signals detectable by using linear dichroism (a differential polarized light spectroscopy technique). A number of methods have been developed to modify chemically the surface of the M13, adding an extensive list of moieties to the surface of the virus.

The M13 naturally possesses different chemical groups at each end [23]. Therefore, it is theoretically possible to chemically tether one end to a surface and visualize it under various shear conditions as a nanotuft (Fig. 1). In this work, we report our observations of the behavior of M13 in fluid flow together with the chemical modification protocols developed in our laboratory [22] to produce a system that can be used to study flow at surfaces. It results in a simple, novel, scalable, synthetic biology method for measuring WSS on a sub-micron scale by using fluorescent microscopy. The nanosensor is an M13 with a specific binding agent (e.g. an antigen-specific antibody) attached to 


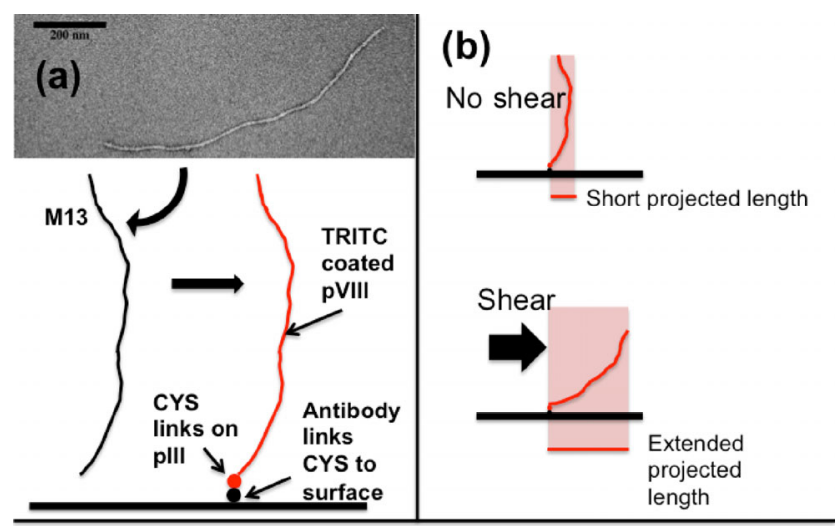

(c)

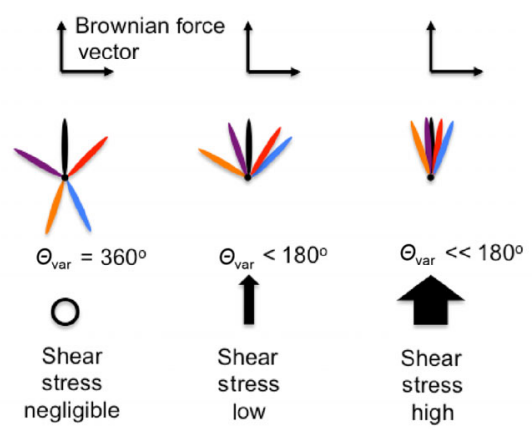

Figure 1 Principles and design of bacteriophage construct. (a) Transmission electron micrograph of M13 with uranyl acetate stain. A schematic of the M13 followed by our process of using cysteines (CYS) on the pIII proteins to bind a molecule designed to affix the M13 to a target surface. pVIII proteins are covalently labeled with a fluorescent isothiocyanate derivative TRITC for fluorescent imaging. (b) A possible method of detection by length. (c) A possible method of detection by variation in angle.

one end via pIII proteins and fluorophores attached to the pVIII proteins (Fig. 1). The appropriate antigen was attached to the surface of the flow cell and its behavior visualized in flow. Subsequent data analysis allowed us to determine the WSS for both the collagen and cultured endothelial cells affixed to coated plastic slides. The result is a nanosensor that is potentially usable in vivo.

\section{Experimental}

Wheat germ agglutinin (WGA) was obtained from Sigma-Aldrich, Poole, UK and the anti-collagen antibody from Abcam, Cambridge, UK. Other chemicals were obtained from the suppliers given in the Electronic Supplementary Material (ESM) and used as received unless stated otherwise. M13 were grown, purified, and fluorescently labeled as described in detail elsewhere [22] and outlined in the ESM. M13 was derivatized with the protein anchor attached to the pIII protein as described in the ESM. The structure of the nanosensor construct is schematically illustrated in Fig. 1(a). Collagen IV-coated microchannel plates ( $\mu$-Slide I ${ }^{0.8}$ Luer, ibidi, Munich) were assembled into a controlled flow system by using a pump (ibidi, Munich). We have previously shown that ibidi systems are able to orient semi-rigid molecular systems [24]. Human conditionally immortalized glomerular endothelial cells (GEnCs) [25] were grown as described in the ESM. The completed M13 construct was bound to the flow slide coating (collagen IV or endothelial cells) and any unbound M13 was rinsed off.

A nominal WSS (0-3.5 dyn $\left.\cdot \mathrm{cm}^{-2}\right)$ was applied. See ESM and the ibidi website for further details. The fluorescent images of the derivatized and labeled M13 construct in flow were measured by using either a spinning disk confocal microscope (Ultraview spinning disk confocal microscope with a 1.4 NA, $100 \times$ oil immersion objective, $561 \mathrm{~nm}$ laser, tetramethylrhodamine5-(and-6)-isothiocyanate (TRITC) filter sets (Croma), and an ORCA $\mathrm{R}^{2}$ camera (Hamamatsu) under control of Volocity 6.3, Perkin-Elmer) at 10 frames per second or a Nikon Ti Eclipse inverted microscope through a $60 \times$ oil immersion objective 1.4 (Nikon Plan Apo VC 60× Oil DIC N2).

Recorded time-lapse images of collagen-coated slides were post-analyzed by using a bespoke function in MATLAB 2014b (MATLAB R2014b, The MathWorks Inc., Natick, MA). Videos and more details are available in the ESM.

From our analysis, we determined the orientation angle and approximate length and width of the M13 nanosensor in each frame. Using MATLAB, the region that the M13 occupies on each frame was first defined by finding a threshold pixel intensity below which all pixels were set to zero and above which they were set to one (Otsu method [26]). The pixels with value one whose nearest neighbor also had value one were then defined as regions of interest (ROI). The largest of these was assumed to be the M13. An image stack with the largest ROI subtracted out was created to be the background and was subtracted from the original binary image, leaving only a M13 nanosensor 
in each image. To smooth the edges of this ROI and fill any pixels of zero intensity in its interior - both of which would affect subsequent analysis - a convex polygon was fitted to the ROI. The major axis was then defined as the longest possible straight line that could be drawn within the ROI, and its angle, determined relative to the horizontal axis of the image, defined the bacteriophage's orientation. The directionality plugin in Fiji/ImageJ [27] gave comparable results.

The tethered nanoparticle motion under Brownian effects and flow was modeled phenomenologically by a random walk with spatially homogeneous jump probability, but a spatially-varying difference in the probability of left or right jumps, modeling the biasing effect of the viscous restoring force as described in the ESM.

\section{Results and discussion}

\subsection{Production and microscopy of labeled and derivatized nanosensors}

Two systems were developed using a novel M13 nanosensor to determine WSS.

(i) Fluorescent M13 constructs derivatized with anticollagen IV antibodies (M13-aCol-TRITC) attached to a collagen IV-coated microchannel plate substrate. The collagen IV was designed to act as a relatively flat surface to test the principles of the nanosensor.

(ii) Fluorescent M13 constructs derivatized with WGA (M13-WGA-TRITC) attached to cultured endothelial cells (GEnCs) via the glycocalyx (WGA binds to sialic acid residues on the surface of glycoproteins that form the glycocalyx on most endothelial cells). The endothelial cells were designed to act as a model blood vessel.

\subsection{Detection of WSS on collagen coated slides}

Spinning disc images of M13-aCol-TRITC exposed to a range of nominal WSS (0-3.5 dyn $\left.\cdot \mathrm{cm}^{-2}\right)$ were collected. The data from these experiments take the form of time-lapse images of individual M13-aCol-TRITC constructs on the surface. The constructs could be recognized under the microscope as bright points, agitating in the buffer. Once under high digital magnification, the M13-aCol-TRITC constructs appear as elliptical shapes, owing to the diffraction limit of light, that adopt different angles as a function of time (Fig. 2(a)). Fourteen independent M13-aCol-TRITC data sets were collected at each flow condition. Once an M13-aCol-TRITC construct was in focus, the flow was induced to test if the construct was bound. Images of the flow slide with no shear flow applied suggested that the M13-aCol-TRITC construct is on the surface and oriented in a random fashion (in response to Brownian motion, Fig. 2(c)). The reaction to flowinduced shear is obvious by eye: The fluorescent ellipse orients, seemingly instantaneously, with its long axis preferentially aligned with the shear flow direction. The degree of orientation increases with flow rate. The microscope focus typically required adjustment to achieve optimal imaging. The signal to noise decreased over time as would be expected if bleaching was occurring; however, more than $10 \mathrm{~min}$ of continuous imaging was possible with both the spinning disk confocal and wide-field microscopes. An example of a M13-aCol-TRITC video (Movie S1) is available in the ESM.

\subsection{Measurement of WSS on GEnCs}

GEnCs, normally used as a model capillary system for kidney disease, were seeded onto sterile, collagencoated flow slides. These kidney-derived cells provide an intellectual bridge to future work on how complications of diabetes (e.g. nephropathy) affects flow in kidneys. WGA conjugated to the PIII protein of M13 (M13-WGA-TRITC) made a body that was visualized by using a wide-field fluorescent microscope showing that the bacteriophage had successfully adhered to cells on the flow slide surface (Fig. 2(b)) and had formed a confluent layer.

Time-lapse images show that in the absence of flow the conjugates move fairly randomly in response to Brownian motion in a manner consistent with attachment to the cellular surface at only one end. The angle space it explores is reduced compared with the collagen-only slides (Figs. 2(c) and 2(d)) and the mean angles in both negative and positive flow directions are non-zero, in contrast to the collagen surfaces. This suggests the bacteriophage is located 

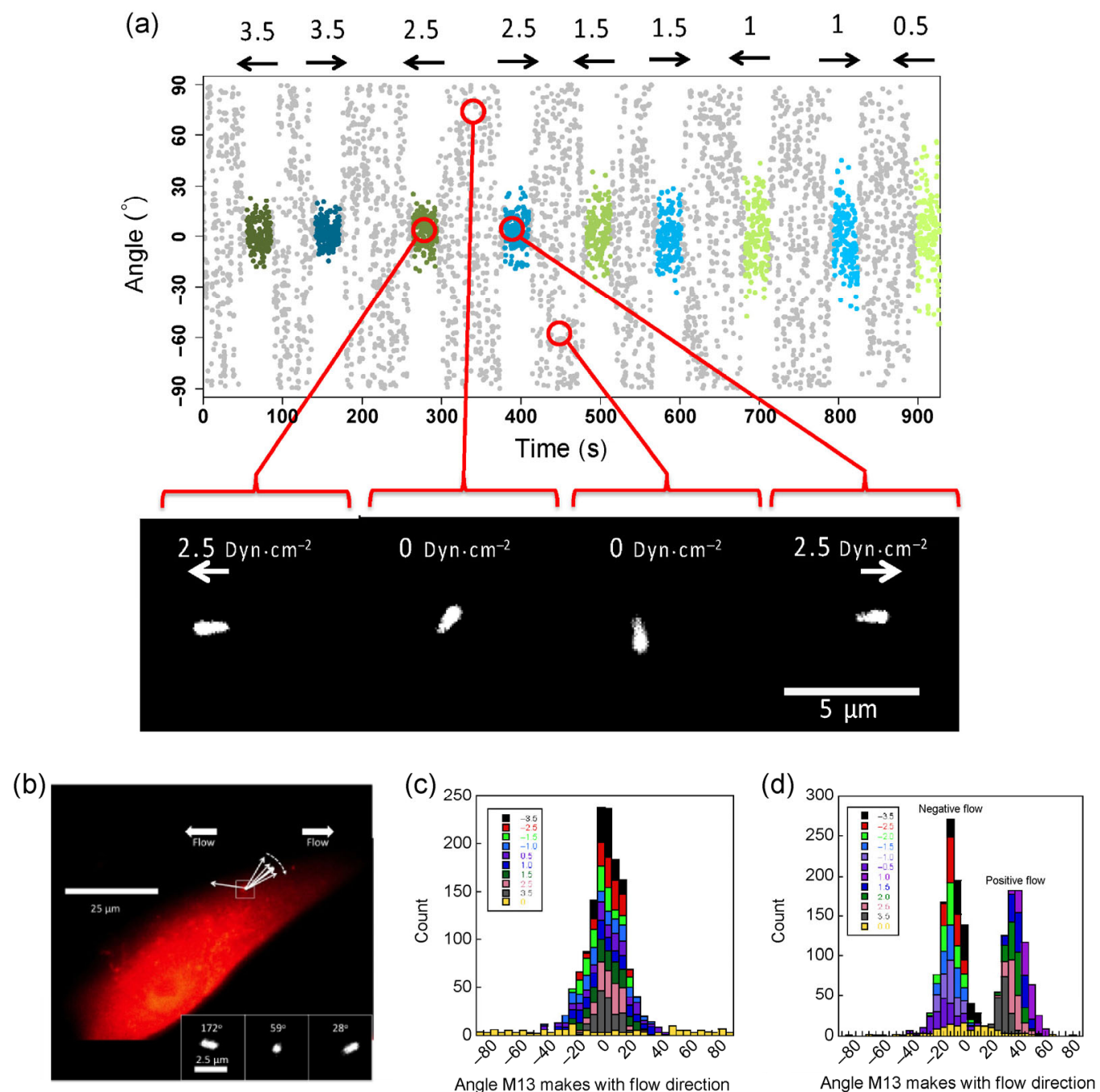

Figure 2 (a) Example of wall shear stress visualized by M13-aCol-TRITC bound to a collagen IV coated flow slide. M13 orientation vs. time in a typical experiment. The nominal wall shear stress (assuming a linear relationship between bulk fluid flow and WSS) is indicated at the top in dyn $\mathrm{cm}^{-2}$. The flow direction and magnitude at each time point are indicated by the arrows and by the color and intensities of the overlays (negative (blue), positive (green), and zero (grey)). Example images at time points corresponding to the red circles in background subtracted spinning disk confocal image frames are shown below. (b) Wall shear stress effect on a M13WGA-TRITC bound to the surface of a cultured GEnC demonstrated by inset snapshots of three flows, giving the indicated nominal wall shear stress and the average orientation. Additive histograms for angles made by a bound M13 with (c) a collagen-only coated slide and (d) a surface layered with GEnCs.

on the side of a cell, near the top, with the cell surface forcing minimum angles of approximately $-10^{\circ}$ for negative flow and $+30^{\circ}$ for positive flow.

\subsection{Quantitation of response of M13-aCol-TRITC construct to flow and WSS}

To use the above data to quantify WSS, a robust data analysis methodology was required. This has two parts.
3.4.1 Turning the videos into orientation data of the type summarized in Fig. 2(a)

MATLAB code was written to extract the bacteriophage orientation and major axis length data. Its angle relative to the horizontal axis of the image defined the bacteriophage's orientation as summarized in Figs. 2(a) and 2(c). The MATLAB segmentation code was found to be accurate by eye and gave similar results to the ImageJ directionality plugin. A video comparison is 
shown in Movie S2 (in the ESM). Both methods gave erratic readings when there was poor focus or other poor signal-to-noise-ratio situations. Moreover, if the M13 appeared round (due to pointing straight up) the angles derived were erratic by both methods.

\subsubsection{Development of a theoretical model of how the bacteriophage orientation relates to the flow}

Both the apparent mean length of the bacteriophage and the variation of its orientation in flow will depend on the shear stress it experiences. If a theoretical model is to be of any use, it must be consistent with the experimental data. The maximum mean length in most examples was $\sim 1.3 \mu \mathrm{m}$, which is consistent with the expected M13 length plus the expected Airy disk, indicating the M13 is almost parallel to the surface at
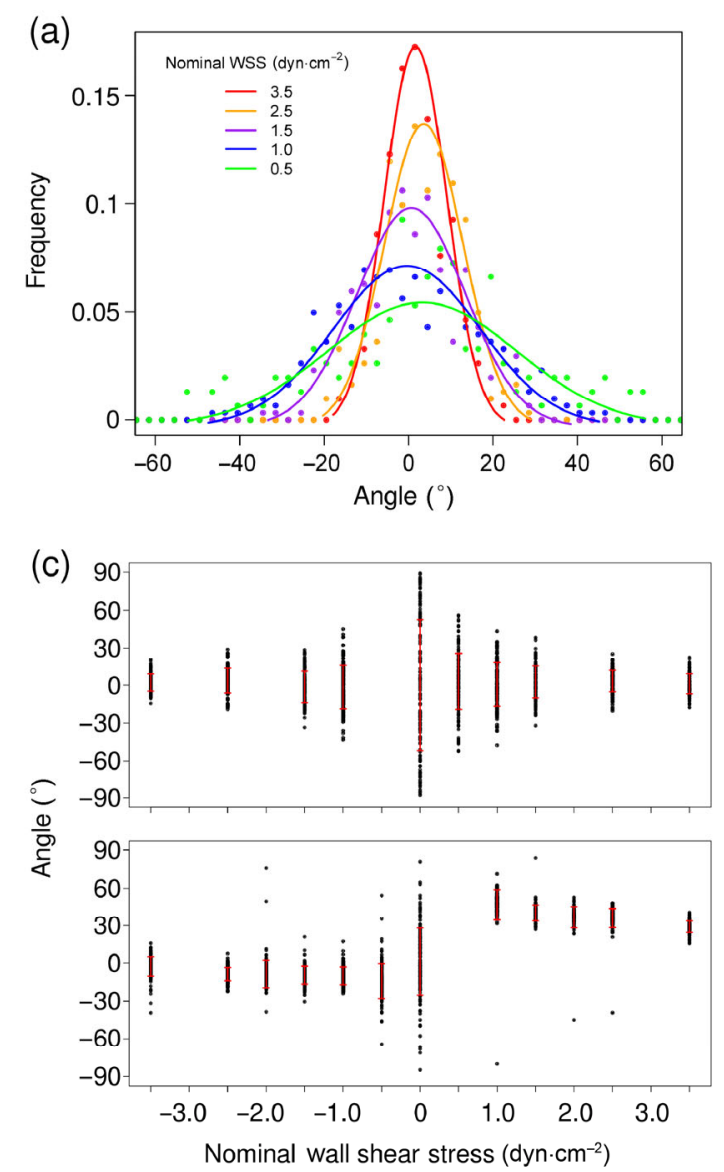

maximum flow and the measured length decreased with flow velocity. There was a correlation between length and nominal WSS; however, as there are only a few pixels between maximum and minimum lengths the resolution was too low for WSS measurement. Fortunately, the variation in angle showed more resolution.

A simple two-dimensional random walk model predicts a normal distribution of angle about a mean direction with the logarithm of the standard deviation of the angle $(\sigma)$ being linearly related to the logarithm of the WSS, with a slope of 0.5 (see ESM)

$$
\ln (\sigma)=-0.5 \ln (\mathrm{WSS})-1.5 \ln (L)+k
$$

where $L$ is the particle length and $k$ is a constant. The data in Figs. 3(a) (Run A) and 3(b) (collagen combined
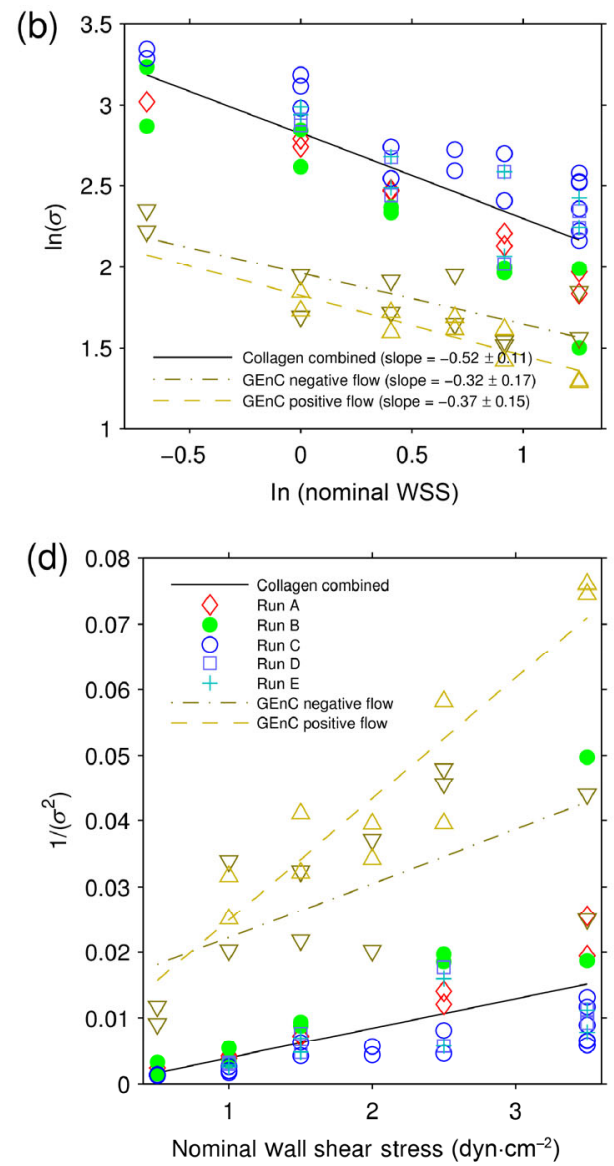

Figure 3 (a) The normalized frequency distribution of angles for an M13 bacteriophage anchored to a collagen coated slide with a Gaussian fit to the original data points (Run A in (b)) as a function of nominal-flow-induced-WSS in dyn $\cdot \mathrm{cm}^{-2}$. (b) $\ln (\sigma) \mathrm{vs}$. $\ln (\mathrm{nominal}$ WSS) for 5 measured M13-aCol-TRITC movies and one GEnC movie all involving both positive and negative direction flows, for $\sigma$ the standard deviation of the angle at each flow rate. (c) Orientation vs. nominal WSS for an M13 anchored to collagen (top) and GEnC coated slides (bottom), negative flow (left), positive flow (right). $\sigma$ is denoted by the bold red bar for each flow. (d) Plots of inverse variance vs. nominal WSS for the data plotted in (b). Quoted errors on the slopes are 95\% confidence intervals of the fit to a straight line. 
data) for 5 independent runs of an M13 bacteriophage anchored onto a collagen-coated flat slide were in accord with such a model, where WSS on the horizontal axis was a nominal value calculated from bulk flow rates (see ESM). The GEnC data (Fig. 3(a)) were also consistent with the model within the larger experimental error of these experiments. Better statistics, either from a higher frame rate, longer runs, or more bacteriophage would be desirable.

The scatter plots of Fig. 3(c) show clearly the different behavior of the flat collagen coated slides and the two opposite flow directions of the GEnC coated slides: The flow direction has no significant effect on the average M13-aCol-TRITC angle or the standard deviation of its observed angles for the collagen coated slides but has a significant effect on both the average angle and standard deviation of angles of the GEnC slides. The reduced angle space of the GEnC data sets (Fig. 2(d)) may suggest some degree of nonspecific interaction of M13 with the cell surface. However, the space sampled in zero flow is still bigger than at the lowest flow rates, so these interactions will not significantly affect the analysis.

Although the $x$-intercepts of the $\ln / \ln$ plots of Fig. 3(b) contain the information to determine the true WSS from $\ln (\sigma)$ and $\ln$ (nominal WSS), the error in the intercept is large. So instead Eq. (3) was plotted.

$$
\frac{1}{\sigma^{2}} \propto \text { nominal WSS }
$$

Nominal WSS is the value of the WSS if the bulk flow is a correct predictor of the flow at the surface. We expect this to be accurate for the collagen experiments. By way of contrast, even though the slopes of the two GEnC lines in Fig. 3(d) have a large error, they are clearly larger than those of the collagen-coated slides, indicating that the bulk-flow-determined nominal WSS is not a good estimate of WSS at the cell surface. The slopes of Fig. 3(d) suggest that the negative flow direction has WSS of more than twice the nominal value and the positive direction over three times the nominal value.

The $\mu$-slide is approximately $80 \times$ deeper than the cell so the enhanced WSS of the GEnC experiments is unlikely to be from channel narrowing. Various other factors presumably contribute to the observation, including roughness of the surface, local viscosity effects, and the shape of the protrusion into the $\mu$-slide. Barbee et al. [28] measured the height of endothelial cells above a surface by using atomic force microscopy and approximated the shear stress due to flow across the surface through a simplified computational fluid dynamics approach: Their result suggests approximately $50 \%$ enhancement of WSS due to the shape protrusion of the cells. Pozrikidis [29] mathematically modeled flow over protuberances on a plane wall: For an elongated hemispheroid with an aspect ratio of 0.2 (similar to the [28] cells) WSS was calculated to increase by $1.5 \times$; for an aspect ratio of 0.5 this factor increased to $2.5 \times$. Thus, much of the increase in WSS with our GEnC experiments is due to the shape of the cells compared with the flat collagen slides; however, other factors are also involved and show the importance of being able to measure WSS locally.

\section{Conclusions}

WSS is usually calculated by using flow velocity gradients measured close to the wall with boundaries and Newtonian assumptions that are often approximations. Here, we have shown that by anchoring one end of a fluorescent M13 bacteriophage onto a surface, the free body of the nanoreporter repositions itself in response to directional flow, resulting in a novel nanosensor that can be used to measure WSS with a $1 \mu \mathrm{m}$ spatial resolution, which is $100 \times$ better than currently implemented methods.

We calibrated the approach with a model system to demonstrate that we could predict the relationship, by using a simple theoretical model, between particle flow behavior and WSS. We were then able to measure local WSS of the irregular surface of a cultured cell, proving the concept and showing that the normal predictions by bulk flow cannot be relied upon. The system is adaptable and can in principle be used in in vivo systems, as it requires only wide-field fluorescent microscopy. The technique and production methodology have been demonstrated on two types of surfaces: relatively flat collagen-coated slides, which compare well with bulk flow expectations, and an endothelial cell-coated surface, which show higher WSS than bulk flow values. Our data therefore suggest 
that WSS operative in physiological systems is likely to deviate significantly from that expected on the basis of bulk flow estimates.

There are several key areas to develop, none of which we believe are insurmountable, before the technique can be used in vitro or in vivo to explore how differences in cell surfaces are affected by local flow effects. The physical properties of the nanofibre-cell binding, in particular its compliance, may affect the precise details of the response to shear. However, provided that the fibre is able to undergo relatively free rotation as exhibited in the present data, the balance between Brownian rotation and shear flow deflection will provide a sensitive and accurate indication of WSS once calibrated to the particular construct.

In summary, although further construct and dataquality developments are needed, this bacteriophage construct, with low manufacturing costs and high adaptability, suggests a way to map flow and WSS on a micron scale in biological systems, thus opening up a wide range of potential applications in fundamental research and diagnosis.

\section{Acknowledgements}

The technical expertise of Ian Hands-Portman at the Imaging Suite, of Life Sciences, University of Warwick, Coventry, CV4 7AL contributed significantly to this work. The funding from a variety of sources to support this work is gratefully acknowledged: Engineering and Physical Sciences Research Council (Impact Acceleration Account EP/K503873/1: M. J. S., K. P. A., T. R. D., M. R. H.); European Union FP7 Marie Curie Initial Training Network (Innovative Doctoral Programme: D. P. L., A. R.); The Science City Research Alliance (K. P. A.); Bizkaia Talent Fellowship (No. AYD-000-256); Engineering and Physical Sciences Research Council (MOAC Doctoral Training Centre, No. EP/F500378/1: A. W.); Medical Research Council (No. G0802829: A. H. J. S., K. B. B.); British Heart Foundation (No. FS/10/017/28249: R. R. F.; No. FS/13/42/30377: A. S.; No. PG/15/37/31438: K. P. A., D. O. B., T. R. D.) and the Libyan higher education ministry (No. 9139, H. E. E.).

Electronic Supplementary Material: Supplementary material (protocols, the basis of the theoretical analysis used, and time-lapses of the figures) is available in the online version of this article at http://dx.doi.org/ 10.1007/s12274-015-0831-x.

\section{References}

[1] Nge, P. N.; Rogers, C. I.; Woolley, A. T. Advances in microfluidic materials, functions, integration, and applications. Chem. Rev. 2013, 113, 2550-2583.

[2] Zarins, C. K.; Giddens, D. P.; Bharadvaj, B. K.; Sottiurai, V. S.; Mabon, R. F.; Glagov, S. Carotid bifurcation atherosclerosis. Quantitative correlation of plaque localization with flow velocity profiles and wall shear-stress. Circ. Res. 1983, 53, 502-514.

[3] Chatzizisis, Y. S.; Coskun, A. U.; Jonas, M.; Edelman, E. R.; Feldman, C. L.; Stone, P. H. Role of endothelial shear stress in the natural history of coronary atherosclerosis and vascular remodeling: Molecular, cellular, and vascular behavior. J. Am. Coll. Cardiol. 2007, 49, 2379-2393.

[4] Katritsis, D.; Kaiktsis, L.; Chaniotis, A.; Pantos, J.; Efstathopoulos, E. P.; Marmarelis, V. Wall shear stress: Theoretical considerations and methods of measurement. Prog. Cardiovasc. Dis. 2007, 49, 307-329.

[5] Reneman, R. S.; Hoeks, A. P. G. Wall shear stress as measured in vivo: Consequences for the design of the arterial system. Med. Biol. Eng. Comput. 2008, 46, 499-507.

[6] Reneman, R. S.; Arts, T.; Hoeks, A. P. G. Wall shear stressan important determinant of endothelial cell function and structure-in the arterial system in vivo. J. Vasc. Res. 2006, 43, 251-269.

[7] Young, E. W. K.; Beebe, D. J. Fundamentals of microfluidic cell culture in controlled microenvironments. Chem. Soc. Rev. 2010, 39, 1036-1048.

[8] Naughton, J. W.; Sheplak, M. Modern developments in shear-stress measurement. Prog. Aerosp. Sci. 2002, 38, 515-570.

[9] Große, S.; Schröder, W. Mean wall-shear stress measurements using the micro-pillar shear-stress sensor MPS3. Meas. Sci. Technol. 2008, 19, 015403.

[10] Brücker, C.; Spatz, J.; Schröder, W. Feasability study of wall shear stress imaging using microstructured surfaces with flexible micropillars. Exp. Fluids. 2005, 39, 464-474.

[11] Smith, M. L.; Long, D. S.; Damiano, E. R.; Ley, K. Nearwall $\mu$-PIV reveals a hydrodynamically relevant endothelial surface layer in venules in vivo. Biophys. J. 2003, 85, 637-645.

[12] Samady, H.; Eshtehardi, P.; McDaniel, M. C.; Suo, J.; Dhawan, S. S.; Maynard, C.; Timmins, L. H.; Quyyumi, A. A.; Giddens, D. P. Coronary artery wall shear stress is associated with 
progression and transformation of atherosclerotic plaque and arterial remodeling in patients with coronary artery disease. Circulation 2011, 124, 779-788.

[13] Mao, C. B.; Solis, D. J.; Reiss, B. D.; Kottmann, S. T.; Sweeney, R. Y.; Hayhurst, A.; Georgiou, G.; Iverson, B.; Belcher, A. M. Virus-based toolkit for the directed synthesis of magnetic and semiconducting nanowires. Science 2013, 303, 213-217.

[14] Murugesan, M.; Abbineni, G.; Nimmo, S. L.; Cao, B. R.; Mao, C. B. Virus-based photo-responsive nanowires formed by linking site-directed mutagenesis and chemical reaction. Sci. Rep. 2013, 3, 1820.

[15] Nam, K. T.; Kim, D. W.; Yoo, P. J.; Chiang, C. Y.; Meethong, N.; Hammond, P. T.; Chiang, Y. M.; Belcher, A. M. Virusenabled synthesis and assembly of nanowires for lithium ion battery electrodes. Science 2006, 312, 885-888.

[16] Chiang, C. Y.; Mello, C. M.; Gu, J.; Silva, E. C. C. M.; Van Vliet, K. J.; Belcher, A. M. Weaving genetically engineered functionality into mechanically robust virus fibers. $A d v$. Mater. 2007, 19, 826-832.

[17] Niu, Z. W.; Bruckman, M. A.; Harp, B.; Mello, C. M.; Wang, Q. Bacteriophage M13 as a scaffold for preparing conductive polymeric composite fibers. Nano Res. 2008, 1, 235-241.

[18] Domaille, D. W.; Lee, J. H.; Cha, J. N. High density DNA loading on the M13 bacteriophage provides access to colorimetric and fluorescent protein microarray biosensors. Chem. Commun. 2013, 49, 1759-1761.

[19] Suthiwangcharoen, N.; Li, T.; Li, K.; Thompson, P.; You, S. J.; Wang, Q. M13 bacteriophage-polymer nanoassemblies as drug delivery vehicles. Nano Res. 2011, 4, 483-493.

[20] Carrico, Z. M.; Farkas, M. E.; Zhou, Y.; Hsiao, S. H.; Marks, J. D.; Chokhawala, H.; Clark, D. S.; Francis, M. B. $\mathrm{N}$-terminal labelling of filamentous phage to create cancer marker imaging agents. ACS Nano 2012, 6, 6675-6680.

[21] Khalil, A. S.; Ferrer, J. M.; Brau, R. R.; Kottmann, S. T.; Noren, C. J.; Lang, M. J.; Belcher, A. M. Single M13 bacteriophage tethering and stretching. Proc. Nat. Acad. Sci. USA 2007, 104, 4892-4897.

[22] Pacheco-Gómez, R.; Kraemer, J.; Stokoe, S.; England, H. J.; Penn, C. W.; Stanley, E.; Rodger, A.; Ward, J.; Hicks, M. R.; Dafforn, T. R. Detection of pathogenic bacteria using a homogeneous immunoassay based on shear alignment of virus particles and linear dichroism. Anal. Chem. 2012, 84, 91-97.

[23] Sidhu, S. S. Engineering M13 for phage display. Biomol. Eng. 2001, 18, 57-63

[24] Cheng, X.; Joseph, M. B.; Covington, J. A.; Dafforn, T. R.; Hicks, M. R.; Rodger, A. Continuous-channel flow linear dichroism. Anal. Methods 2012, 4, 3169-3173.

[25] Satchell, S. C.; Tasman, C. H.; Singh, A.; Ni, L.; Geelen, J.; von Ruhland, C. J.; O'Hare, M. J.; Saleem, M. A.; van den Heuvel, L. P.; Mathieson, P. W. Conditionally immortalized human glomerular endothelial cells expressing fenestrations in response to VEGF. Kidney Int. 2006, 69, 1633-1640.

[26] Otsu, N. A threshold selection method from gray-level histogram. IEEE Trans. Syst. Man Cybernetics. 1979, 9, 62-66.

[27] Schindelin, J.; Arganda-Carreras, I.; Frise, E. Kaynig, V.; Longair, M.; Pietzsch, T.; Preibisch, S.; Rueden, C.; Saalfeld, S.; Schmid, B. et al. Fiji: An open-source platform for biological-image analysis. Nat. Methods 2012, 9, 676-682.

[28] Barbee, K. A.; Mundel, T.; Lal, R.; Davies, P. F. Subcellular distribution of shear stress at the surface of flow-aligned and nonaligned endothelial monolayers. Am. J. Physiol. 1995, 268, H1765-H1772.

[29] Pozrikidis, C. Shear flow over a protuberance on a plane wall. J. Eng. Math. 1997, 31, 29-42.

[30] Arkill, K. P.; Neal, C. R.; Mantell, J. M.; Michel, C. C.; Qvortrup, K.; Rostgaard, J.; Bates, D. O.; Knupp, C.; Squire, J. M. 3D reconstruction of the glycocalyx structure in mammalian capillaries using electron tomography. Microcirculation 2012, 19, 343-351. 